\title{
ROBUST OUTPUT REGULATION OF SINGULAR NONLINEAR SYSTEMS*
}

\author{
ZHIYONG $\mathrm{CHEN}^{\dagger}$ AND JIE HUANG ${ }^{\dagger}$
}

\begin{abstract}
Singular systems, defined as dynamical systems subject to algebraic constraints, arise in many engineering disciplines. The output regulation problem for singular nonlinear systems has been studied recently for the ideal case where the mathematical model is exactly known. This paper will consider the robust output regulation problem for a class of singular nonlinear systems which contain uncertain parameters. We will establish the conditions for the solvability of the problem, thus extending the existing results from normal nonlinear systems to singular nonlinear systems.
\end{abstract}

1. Introduction. Singular systems arise in many areas of engineering including electrical networks, power system, aerospace engineering and chemical processing. Since the late 1970s singular systems have attracted the attention of many researchers. Several books and survey papers dealing with these systems have addressed the issues of solvability, controllability and observability, pole assignment, the elimination of impulsive behavior, and so on [6], [20], [8], [3]. This paper will consider the robust output regulation problem for a class of singular nonlinear systems to be described in Section 2. Briefly, the output regulation problem aims to design control laws for a plant so that the output of the closed-loop system is able to asymptotically track a class of reference inputs and reject a class of disturbances. Both the disturbance and reference are generated by an autonomous differential equation called exosystem. When the controller is also required to tolerate certain plant uncertainty, the problem is called a robust output regulation problem. For linear systems, this problem was thoroughly studied for the normal systems in the 1970s in [10], [11] among others. A salient outcome of these research activities is the internal model principle which is the extension of the well known PID control. The problem was also investigated for linear singular systems in 1980's [8]. Recently, a more clear-cut solution of this problem for linear singular systems was obtained in [21]. For nonlinear systems, the same problem was first treated for normal systems. The special case in which the exogenous signals are constant was studied in [11], [16]. The general case with time varying exogenous signals was studied in [19] without considering the parameter uncertainty. Subsequently, the robust version of the same problem was pursued in [13], [14], [12], [2]. More recently, the output regulation problem for singular nonlinear systems was formulated and solved in [18]. The objective of this paper is to extendthe research initiated in [18] by considering the plant uncertainty.

*Invited paper. Received on October 14, 2001; accepted for publication on January 14, 2002. The work described in this paper was partially supported by a grant from the Research Grant Council of the Hong Kong Special Administrative Region (Project No. CUHK4168/98E).

${ }^{\dagger}$ Department of Automation and Computer-Aided Engineering, The Chinese University of Hong Kong, Shatin, N.T., Hong Kong, Email: zychen@acae.cuhk.edu.hk, jhuang@acae.cuhk.edu.hk 
The paper is organized as follows: Section 2 describes the robust output regulation problem for singular systems and summarizes some results obtained in [18]. In section 3 , we give a preliminary result which solves the $k^{t h}$-order robust output regulation problem. The main result of this paper is given in section 4, where we will derive the solvability condition for the problem, and construct the controller. The result can be viewed as a generalization of the same problem for the normal nonlinear systems as in [12]. Finally, section 5 concludes this paper.

2. Problem description and standard assumptions. Consider the plant described by

$$
\left\{\begin{array}{l}
\mathbf{E} \dot{x}(t)=f(x(t), u(t), v(t), w), x(0)=x_{0} \\
y(t)=h(x(t), v(t), w), t \geq 0
\end{array}\right.
$$

and an exosystem described by

$$
\dot{v}(t)=a(v(t)), v(0)=v_{0}
$$

where $x(t) \in \Re^{n}$ is the plant state, $u(t) \in \Re^{m}$ the plant input, $y(t) \in \Re^{p}$ the plant output representing the tracking error, $v(t) \in \Re^{q}$ the exogenous signal representing the disturbance and/or the reference input, $w \in \Re^{N}$ the plant unknown parameters, and $\mathbf{E} \in \Re^{n \times n}$ a singular constant matrix, and $\operatorname{rank}(\mathbf{E})=n_{E}<n$. It is noted that if $n_{E}=n$, the plant is called normal. Also it is assumed that the nominal value of the uncertain parameters $w$ is 0 .

This paper will focus on the dynamic output feedback controller as follows:

$$
\left\{\begin{array}{l}
u(t)=k(z(t), y(t)) \\
\dot{z}(t)=g(z(t), y(t))
\end{array}\right.
$$

where $z(t)$ is the compensator state vector of dimension $n_{c}$. When the state of the system is available, the state feedback control law can be considered accordingly.

The closed-loop system composed of plant (2.1), (2.2) and control law (2.3) can be put into the following form:

$$
\left\{\begin{array}{l}
\mathbf{E}_{c} \dot{x}_{c}(t)=f_{c}\left(x_{c}(t), v(t), w\right), x_{c}(0)=x_{c 0} \\
y(t)=h_{c}\left(x_{c}(t), v(t), w\right)
\end{array}\right.
$$

where

$$
\left\{\begin{array}{l}
x_{c}=\left[\begin{array}{l}
x \\
z
\end{array}\right], \mathbf{E}_{c}=\left[\begin{array}{cc}
\mathbf{E} & 0 \\
0 & I_{n_{c}}
\end{array}\right] \\
f_{c}\left(x_{c}, v, w\right)=\left[\begin{array}{c}
f(x, k(z, h(x, v, w)), v, w) \\
g(z, h(x, v, w))
\end{array}\right] \\
h_{c}\left(x_{c}, v, w\right)=h(x, v, w)
\end{array}\right.
$$

Throughout this paper, it is assumed that all the functions involved in this setup are sufficiently smooth and defined globally on the appropriate Euclidean spaces, and 
$a(0)=0, f(0,0,0, w)=0$, and $h(0,0, w)=0$ for any $w \in W$ with $W$ an open neighborhood of the origin of $\Re^{N}$. Our results will be stated locally in terms of $V$ and $W$ with $V$ an open neighborhood of the origin in $\Re^{q}$. In the sequel, $V$ and $W$ are implicitly permitted to be made smaller to accommodate subsequent local arguments.

The linearization of the system $(2.1)$ and $(2.2)$ at $(x, u, v)=(0,0,0)$ will be frequently used, therefore, the following notation is given,

$$
\begin{gathered}
A(w)=\left.\frac{\partial f}{\partial x}\right|_{x=0, u=0, v=0}, B(w)=\left.\frac{\partial f}{\partial u}\right|_{x=0, u=0, v=0}, \\
E(w)=\left.\frac{\partial f}{\partial v}\right|_{x=0, u=0, v=0}, C(w)=\left.\frac{\partial h}{\partial x}\right|_{x=0, v=0}, \\
F(w)=\left.\frac{\partial h}{\partial v}\right|_{x=0, v=0}, A_{1}=\left.\frac{\partial a(v)}{\partial v}\right|_{v=0}, A_{c}(w)=\left.\frac{\partial f_{c}}{\partial x_{c}}\right|_{x_{c}=0, v=0}
\end{gathered}
$$

As a result, the system (2.1) and (2.2) can also be written as

$$
\left\{\begin{array}{l}
\mathbf{E} \dot{x}=A(w) x+B(w) u+E(w) v+o(x, u, v, w) \\
y=C(w) x+F(w) v+o(x, u, v, w) \\
\dot{v}=A_{1} v+o(v)
\end{array}\right.
$$

where $o(x, u, v, w)$ (or $o(v))$ is a sufficiently smooth function vanishing at $(x, u, v)=$ $(0,0,0)$ (or $v=0$ ) together with its first order derivative for any $w \in W$. For convenience, let $A, B, \cdots$, denote $A(0), B(0), \cdots$, respectively.

The robust output regulation problem: Find a control law such that the closed-loop (2.4) has the two properties:

(P1) the linearization at $x_{c}=0$ of

$$
\mathbf{E}_{c} \dot{x}_{c}(t)=f_{c}\left(x_{c}(t), 0,0\right)
$$

is strongly stable, that is, $\operatorname{deg}\left(\operatorname{det}\left(\lambda \mathbf{E}_{c}-A_{c}\right)=\operatorname{rank}\left(\mathbf{E}_{c}\right)\right.$, and $\sigma\left(\mathbf{E}_{c}, A_{c}\right) \in c^{-}$, where $\sigma\left(\mathbf{E}_{c}, A_{c}\right) \triangleq\left\{\lambda \mid \operatorname{det}\left(\lambda \mathbf{E}_{c}-A_{c}\right)=0\right\}$.

(P2) the trajectories starting from all sufficiently small initial states $\left(x_{c 0}, v_{0}\right)$ satisfy:

$$
\lim _{t \rightarrow \infty} y(t)=\lim _{t \rightarrow \infty} h_{c}\left(x_{c}(t), v(t), w\right)=0, \forall w \in W
$$

REMARK 2.1. The above problem is clearly an extension of the problem studied in [18], taking into account the uncertainty. Viewing $w$ as being generated by an exosystem of the form $\dot{w}=0$, a solvability condition can be obtained by slightly modifying Lemma 4.1 of [18], as follows:

TheOREM 2.2. Assume the following,

A1: The equilibrium of exosystem (2.2) is stable and all the eigenvalues of $(\partial a / \partial v)(0)$ have zero real parts. 
Then the controller (2.3) solves the robust output regulation problem of the singular uncertain system (2.1) and (2.2) if it is such that the closed-loop system satisfies:

(i) (P1) holds.

(ii) there exists a sufficiently smooth function $\mathbf{x}_{c}(v, w)$ locally defined in $V \times W$ satisfying $\mathbf{x}_{c}(0,0)=0$ and

$$
\left\{\begin{array}{l}
\mathbf{E}_{c} \frac{\partial \mathbf{x}_{c}(v, w)}{\partial v} a(v)=f_{c}\left(\mathbf{x}_{c}(v, w), v, w\right) \\
h_{c}\left(\mathbf{x}_{c}(v, w), v, w\right)=0
\end{array}\right.
$$

To close this section, some standard assumptions will be listed.

A2: $(\mathbf{E}, A, B)$ is strongly stabilizable, i.e., there exists a matrix $K \in \Re^{m \times n}$ such that $(\mathbf{E}, A+B K)$ is strongly stable.

A3: $(\mathbf{E}, A, C)$ is strongly detectable, i.e., there exists a matrix $K \in \Re^{n \times p}$ such that $(\mathbf{E}, A+K C)$ is strongly stable.

A4: There exist sufficiently smooth functions $\mathbf{x}(v, w)$ and $\mathbf{u}(v, w)$ such that $\mathbf{x}(0,0)=0$ and $\mathbf{u}(0,0)=0$ and satisfy, for $v \in V, w \in W$

$$
\left\{\begin{array}{l}
\mathbf{E} \frac{\partial \mathbf{x}(v, w)}{\partial v} a(v)=f(\mathbf{x}(v, w), \mathbf{u}(v, w), v, w) \\
h(\mathbf{x}(v, w), v, w)=0
\end{array}\right.
$$

Remark 2.3. When $\mathbf{E}$ is an identity matrix, Assumptions $\mathbf{A 2}$ and $\mathbf{A 3}$ reduce to exactly the same ones assumed by [19] for the normal systems. Equation (2.8) becomes the so called regulator equations discovered by Isidori and Byrnes.

3. A Preliminary Result. Recall that, in the normal case, the way to handle the robust output regulation problem is much more complicated than the way to handle the output regulation problem. This is because, when there is no uncertainty, the solution of the equation (2.8) or its estimation can be used as a feedforward function to cancel the steady state error output; but this is impossible when the uncertain parameter $w$ is present since the solution of equation (2.8) also depends on $w$ which cannot appear in the control law. As a result, the problem has to be approached with the employment of a nonlinear version of the internal model principle [12], [13], [14], etc. Here this technique will be further extended to the singular nonlinear systems. To begin with, some notations that have been used frequently in [12], [13], [14] are first introduced. For any matrix $M$, define

$$
M^{(0)}=I, M^{(1)}=M, \cdots, M^{(k)}=\underbrace{M \otimes \cdots \otimes M}_{k \text { factors }}, k=1,2, \cdots
$$

where $\otimes$ denotes the Kronecker product. Also let $v^{[l]}$ denote the vector

$$
v^{[l]}=\left[v_{1}^{l}, v_{1}^{l-1} v_{2}, \cdots, v_{1}^{l-1} v_{q}, v_{1}^{l-2} v_{2}^{2}, v_{1}^{l-2} v_{2} v_{3}, \cdots, v_{1}^{l-2} v_{2} v_{q}, \cdots, v_{q}^{l}\right]^{T}
$$

It was shown in [12] that if $v$ satisfies $\dot{v}=A_{1} v$ for some square matrix $A_{1}$, then there exist square matrices $A_{l}, l=2,3, \cdots$, such that

$$
\dot{v}^{[l]}(t)=A_{l} v^{[l]}(t), l=2,3, \cdots
$$


In fact, $A_{l}$ can be explicitly given as

$$
A_{l}=M_{l}\left[\sum_{i=1}^{l} I_{q}^{(i-1)} \otimes A_{1} \otimes I_{q}^{(l-i)}\right] N_{l}
$$

where $M_{l}$ and $N_{l}$ are such that $v^{[l]}=M_{l} v^{(l)}, v^{(l)}=N_{l} v^{[l]}$ and $I_{q}$ denote the $q$ dimensional identity matrix. As in [12], the following autonomous system is called K-fold exosystem.

$$
\left[\begin{array}{c}
\dot{v}^{[1]} \\
\dot{v}^{[2]} \\
\vdots \\
\dot{v}^{[k]}
\end{array}\right]=\left[\begin{array}{cccc}
A_{1} & 0 & \cdots & 0 \\
0 & A_{2} & \cdots & 0 \\
\vdots & \vdots & \vdots & \vdots \\
0 & 0 & \cdots & A_{k}
\end{array}\right]\left[\begin{array}{c}
v^{[1]} \\
v^{[2]} \\
\vdots \\
v^{[k]}
\end{array}\right]
$$

A linear result which will play an important role in establishing the major result is first stated:

Lemma 3.1. Given any square matrix $\hat{A}_{1}$ such that all the eigenvalues of $\hat{A}_{1}$ are on the closed right complex plane, let $\beta_{i} \in \Re^{n_{i} \times n_{i}}, \sigma_{i} \in \Re^{1 \times n_{i}}, i=1, \cdots, r$, for some positive integers $n_{1}, \cdots, n_{r}, r$, satisfy the following

(i) $\beta_{i}$ and $\sigma_{i}$ are controllable, and

(ii) the minimal polynomial of $\hat{A}_{1}$ divides the characteristic polynomial of $\beta_{i}$, let

$$
\begin{gathered}
G_{1}=\text { block diag }\left[\beta_{1}, \cdots, \beta_{r}\right], \\
G_{2}=\text { block diag }\left[\sigma_{1}, \cdots, \sigma_{r}\right]
\end{gathered}
$$

and finally, let matrices $g_{1}$ and $g_{2}$ admit the following form

$$
g_{1}=T\left[\begin{array}{cc}
S_{1} & S_{2} \\
0 & G_{1}
\end{array}\right] T^{-1}, \quad g_{2}=T\left[\begin{array}{c}
S_{3} \\
G_{2}
\end{array}\right]
$$

where $S_{1}, S_{2}, S_{2}$ are any matrices with proper dimensions, and $T$ is a nonsingular matrix. Then, for any matrices $\hat{A}, \hat{B}, \hat{C}, \hat{D}$ with appropriate dimensions, if the matrix

$$
\left[\begin{array}{cc}
\hat{A} & \hat{B} \\
g_{2} \hat{C} & g_{1}+g_{2} \hat{D}
\end{array}\right]
$$

is Hurwitz, then for any $(U, V)$ with proper dimension, the linear matrix equation

$$
\left\{\begin{array}{l}
\phi \hat{A}_{1}=\hat{A} \phi+\hat{B} \theta+U \\
\theta \hat{A}_{1}=g_{1} \theta+g_{2}(\hat{C} \phi+\hat{D} \theta+V)
\end{array}\right.
$$

has a unique solution which satisfies

$$
\hat{C} \phi+\hat{D} \theta+V=0
$$


Proof. Since (3.3) is a Sylvester equation, it follows from the assumptions on matrices $\hat{A}_{1}$ and (3.2) that equation (3.3) has a unique solution. To show (3.4), let $\theta=\left[\hat{\theta}^{T}, \bar{\theta}^{T}\right]^{T}$, where $\bar{\theta}$ has the same dimension as that of $G_{1}$. Then the second equation of (3.3) implies

$$
\bar{\theta} \hat{A}_{1}-G_{1} \bar{\theta}=G_{2} Y
$$

where $Y=\hat{C} \phi+\hat{D} \theta+V$.

Due to the block diagonal structure of $G_{1}$ and $G_{2}$, we can assume $r=1$ without loss of generality. Therefore, we can write $G_{1}$ and $G_{2}$ in the following form:

$$
G_{1}=\left[\begin{array}{ccccc}
0 & 1 & \cdots & 0 & 0 \\
0 & 0 & \cdots & 0 & 0 \\
\vdots & \vdots & \vdots & \vdots & \vdots \\
0 & 0 & \cdots & 0 & 1 \\
-\alpha_{n_{k}} & -\alpha_{n_{k}-1} & \cdots & -\alpha_{2} & -\alpha_{1}
\end{array}\right], G_{2}=\left[\begin{array}{c}
0 \\
0 \\
\vdots \\
0 \\
1
\end{array}\right]
$$

Let $\theta_{j}, j=1, \cdots, n_{k}$, denote the $j^{t h}$ row of $\bar{\theta}$. Then expanding (3.5) gives

$$
\left[\begin{array}{c}
\theta_{1} \hat{A}_{1}-\theta_{2} \\
\theta_{2} \hat{A}_{1}-\theta_{3} \\
\vdots \\
\theta_{n_{k}-1} \hat{A}_{1}-\theta_{n_{k}}+\alpha_{n_{k}} \theta_{1}+\cdots+\alpha_{1} \theta_{n_{k}}
\end{array}\right]=\left[\begin{array}{c}
0 \\
0 \\
\vdots \\
0 \\
Y
\end{array}\right]
$$

Furthermore,

$$
Y=\theta_{1}\left(\hat{A}_{1}^{n_{k}}+\alpha_{1} \hat{A}_{1}^{n_{k}-1}+\cdots+\alpha_{n_{k}} I\right)
$$

Noting that the characteristic polynomial of $G_{1}$ is divisible by the minimal polynomial of $\hat{A}_{1}$ gives $Y=0$.

LEMMA 3.2. Under assumption A1, for any positive integer $k$, let

$$
\hat{A}_{1}=\left[\begin{array}{cccc}
A_{1} & 0 & \cdots & 0 \\
0 & A_{2} & \cdots & 0 \\
\vdots & \vdots & \vdots & \vdots \\
0 & 0 & \cdots & A_{k}
\end{array}\right]
$$

Let a linear controller of the form

$$
\left\{\begin{array}{l}
u=K_{1} z+K_{2} y \\
\dot{z}=g_{1} z+g_{2} y
\end{array}\right.
$$

be given where $g_{1}$ and $g_{2}$ are given as in Lemma 3.1 with $r=p$. Then, if the controller (3.6) makes the pair

$$
\left(\left[\begin{array}{cc}
\mathbf{E} & 0 \\
0 & I_{n_{c}}
\end{array}\right],\left[\begin{array}{cc}
A+B K_{2} C & B K_{1} \\
g_{2} C & g_{1}
\end{array}\right]\right)
$$


strongly stable, then the closed-loop system composed of (2.1), (2.2) and controller (3.6) has the property that there exists a sufficiently smooth function $\mathbf{x}_{c}(v, w)$ locally defined in $V \times W$ satisfying $\mathbf{x}_{c}(0,0)=0$, and

$$
\left\{\begin{array}{l}
\mathbf{E}_{c} \frac{\partial \mathbf{x}_{c}(v, w)}{\partial v} a(v)=f_{c}\left(\mathbf{x}_{c}(v, w), v, w\right) \\
h_{c}\left(\mathbf{x}_{c}(v, w), v, w\right)=O\left(v^{k+1}\right)
\end{array}\right.
$$

where $O\left(v^{k+1}\right)$ is such that $\lim _{v \rightarrow 0} \frac{\left\|O\left(v^{k+1}\right)\right\|}{\|v\|^{k+1}}$ is a finite constant.

Proof. This result will be established by performing the standard coordinate transformation on the closed-loop system. To this end, first note that the closed-loop system composed of (2.1),(2.2) and (3.6) can be written as follows,

$$
\left\{\begin{array}{l}
\mathbf{E} \dot{x}=\left(A(w)+B(w) K_{2} C(w)\right) x+B(w) K_{1} z \\
+\left(E(w)+B(w) K_{2} F(w)\right) v+o(x, u, v, w) \\
\dot{z}=g_{2} C(w) x+g_{1} z+g_{2} F(w) v+o(x, u, v, w) \\
y=C(w) x+F(w) v+o(x, u, v, w) \\
\dot{v}=A_{1} v+o(v)
\end{array}\right.
$$

Let $T_{1}$ and $T_{2}$ be two nonsingular matrices such that $T_{1} \mathbf{E} T_{2}=\left[\begin{array}{cc}I_{n_{E}} & 0 \\ 0 & 0\end{array}\right]$. Let

$$
\begin{gathered}
T_{1} A(w) T_{2}=\left[\begin{array}{ll}
A_{11}(w) & A_{12}(w) \\
A_{21}(w) & A_{22}(w)
\end{array}\right], T_{1} B(w)=\left[\begin{array}{c}
B_{1}(w) \\
B_{2}(w)
\end{array}\right], \\
T_{1} E(w)=\left[\begin{array}{c}
E_{1}(w) \\
E_{2}(w)
\end{array}\right], C(w) T_{2}=\left[\begin{array}{ll}
C_{1}(w) & C_{2}(w)
\end{array}\right], T_{2}^{-1} x=\left[\begin{array}{c}
\bar{x}_{1} \\
\bar{x}_{2}
\end{array}\right]
\end{gathered}
$$

with $A_{11}(w) \in \Re^{n_{E} \times n_{E}}, B_{1}(w) \in \Re^{n_{E} \times m}, E_{1}(w) \in \Re^{n_{E} \times q}, C_{1}(w) \in \Re^{p \times n_{E}}, \bar{x}_{1} \in \Re^{n_{E}}$, and other matrices have proper dimensions.

In terms of $\bar{x}_{1}$ and $\bar{x}_{2}$, the first equation of (3.9) becomes

$$
\left\{\begin{array}{l}
\dot{\bar{x}}_{1}=\bar{A}_{11}(w) \bar{x}_{1}+\bar{A}_{12}(w) \bar{x}_{2}+\bar{B}_{1}(w) K_{1} z+\bar{E}_{1}(w) v+o(x, z, v, w) \\
0=\bar{A}_{21}(w) \bar{x}_{1}+\bar{A}_{22}(w) \bar{x}_{2}+\bar{B}_{2}(w) K_{1} z+\bar{E}_{2}(w) v+o(x, z, v, w)
\end{array}\right.
$$

where

$$
\begin{gathered}
\bar{A}_{i j}(w)=A_{i j}(w)+B_{i}(w) K_{2} C_{j}(w) \\
\bar{B}_{i}(w)=B_{i}(w) \\
\bar{E}_{i}(w)=E_{i}(w)+B_{i}(w) K_{2} F(w), i, j \in\{1,2\}
\end{gathered}
$$


We will first show that $\bar{A}_{22}$ is nonsingular. In fact,

$$
\begin{aligned}
& \operatorname{det}\left(\left[\begin{array}{cc}
T_{1} & 0 \\
0 & I_{n_{c}}
\end{array}\right]\left(\lambda\left[\begin{array}{cc}
\mathbf{E} & 0 \\
0 & I_{n_{c}}
\end{array}\right]-\left[\begin{array}{cc}
A+B K_{2} C & B K_{1} \\
g_{2} C & g_{1}
\end{array}\right]\right)\left[\begin{array}{cc}
T_{2} & 0 \\
0 & I_{n_{c}}
\end{array}\right]\right) \\
&=\operatorname{det}\left(\left[\begin{array}{ccc}
\lambda I_{n_{E}}-\left(A_{11}+B_{1} K_{2} C_{1}\right) & -\left(A_{12}+B_{1} K_{2} C_{2}\right) & -B_{1} K_{1} \\
-\left(A_{21}+B_{2} K_{2} C_{1}\right) & -\left(A_{22}+B_{2} K_{2} C_{2}\right) & -B_{2} K_{1} \\
-g_{2} C_{1} & -g_{2} C_{2} & \lambda I_{n_{c}}-g_{1}
\end{array}\right]\right) \\
&=\operatorname{det}\left(\left[\begin{array}{ccc}
\lambda I_{n_{E}}-\bar{A}_{11} & -\bar{A}_{12} & -\bar{B}_{1} K_{1} \\
-\bar{A}_{21} & -\bar{A}_{22} & -\bar{B}_{2} K_{1} \\
-g_{2} C_{1} & -g_{2} C_{2} & \lambda I_{n_{c}}-g_{1}
\end{array}\right]\right) \\
&(3.11) \quad=\operatorname{det}\left(-\bar{A}_{22}\right) \lambda^{n_{E}+n_{c}+b(\lambda)}
\end{aligned}
$$

where $b(\lambda)$ is a polynomial in $\lambda$ of degree smaller than $n_{E}+n_{c}$. It follows from the strong stability of $(3.7)$ that $\operatorname{det}\left(\bar{A}_{22}\right) \neq 0$. Thus the implicit function theorem guarantees the existence of a unique sufficiently smooth solution of the second equation of (3.10), and this solution has the form

$$
\begin{aligned}
\bar{x}_{2} & =\gamma\left(\bar{x}_{1}, z, v, w\right)=-\bar{A}_{22}^{-1}(w) \bar{A}_{21}(w) \bar{x}_{1}-\bar{A}_{22}^{-1}(w) \bar{B}_{2}(w) K_{1} z \\
& -\bar{A}_{22}^{-1}(w) \bar{E}_{2}(w) v+o\left(\bar{x}_{1}, z, v, w\right)
\end{aligned}
$$

Substituting (3.12) into the first equation of (3.10) and the second and third equations of (3.9) gives a reduced order normal system as follows

$$
\left\{\begin{array}{l}
\dot{\bar{x}}_{1}=\bar{f}_{1 c}\left(\bar{x}_{1}, z, v, w\right)=\hat{A}(w) \bar{x}_{1}+\hat{B}(w) z+\hat{E}(w) v+o\left(\bar{x}_{1}, z, v, w\right) \\
\dot{z}=g_{1} z+g_{2} y \\
y=\bar{h}_{c}\left(\bar{x}_{1}, z, v, w\right)=\hat{C}(w) \bar{x}_{1}+\hat{D}(w) z+\hat{F}(w) v+o\left(\bar{x}_{1}, z, v, w\right)
\end{array}\right.
$$

where

$$
\begin{aligned}
& \hat{A}(w)=\bar{A}_{11}(w)-\bar{A}_{12}(w) \bar{A}_{22}^{-1}(w) \bar{A}_{21}(w) \\
& \hat{B}(w)=\left(\bar{B}_{1}(w)-\bar{A}_{12}(w) \bar{A}_{22}^{-1}(w) \bar{B}_{2}(w)\right) K_{1} \\
& \hat{C}(w)=C_{1}(w)-C_{2}(w) \bar{A}_{22}^{-1}(w) \bar{A}_{21}(w) \\
& \hat{D}(w)=\left(-C_{2}(w) \bar{A}_{22}^{-1}(w) \bar{B}_{2}(w)\right) K_{1} \\
& \hat{E}(w)=\bar{E}_{1}(w)-\bar{A}_{12}(w) \bar{A}_{22}^{-1}(w) \bar{E}_{2}(w) \\
& \hat{F}(w)=F(w)-C_{2}(w) \bar{A}_{22}^{-1}(w) \bar{E}_{2}(w)
\end{aligned}
$$

Next we will show the matrix

$$
\left[\begin{array}{cc}
\hat{A} & \hat{B} \\
g_{2} \hat{C} & g_{1}+g_{2} \hat{D}
\end{array}\right]
$$

is Hurwitz. To this end, let

$$
M_{1}=\left[\begin{array}{ccc}
I_{n_{E}} & -\bar{A}_{12} \bar{A}_{22}^{-1} & 0 \\
0 & I_{n-n_{E}} & 0 \\
0 & 0 & I_{n_{c}}
\end{array}\right], M_{2}=\left[\begin{array}{ccc}
I_{n_{E}} & 0 & 0 \\
-\bar{A}_{22}^{-1} \bar{A}_{21} & I_{n-n_{E}} & -\bar{A}_{22}^{-1} \bar{B}_{2} K_{1} \\
0 & 0 & I_{n_{c}}
\end{array}\right] .
$$


Then

$$
\begin{aligned}
& \operatorname{det}\left(M_{1}\left[\begin{array}{ccc}
\lambda I_{n_{E}}-\bar{A}_{11} & -\bar{A}_{12} & -\bar{B}_{1} K_{1} \\
-\bar{A}_{21} & -\bar{A}_{22} & -\bar{B}_{2} K_{1} \\
-g_{2} C_{1} & -g_{2} C_{2} & \lambda I_{n_{c}}-g_{1}
\end{array}\right] M_{2}\right) \\
= & \operatorname{det}\left(\left[\begin{array}{ccc}
\lambda I_{n_{E}}-\hat{A} & 0 & -\hat{B} \\
0 & -\bar{A}_{22} & 0 \\
-g_{2} \hat{C} & -g_{2} C_{2} & \lambda I_{n_{c}}-\left(g_{1}+g_{2} \hat{D}\right)
\end{array}\right]\right) \\
= & \operatorname{det}\left(-\bar{A}_{22}\right) \operatorname{det}\left(\left[\begin{array}{ccc}
\lambda I_{n_{E}}-\hat{A} & -\hat{B} \\
-g_{2} \hat{C} & \lambda I_{n_{c}}-\left(g_{1}+g_{2} \hat{D}\right)
\end{array}\right]\right)
\end{aligned}
$$

Again it follows from the strong stability of (3.7) that the matrix (3.13) is Hurwitz. Thus, from the center manifold theorem [5], there exists sufficiently smooth functions $\overline{\mathbf{x}}_{1}^{(k)}(v, w)$ and $\mathbf{z}^{(k)}(v, w)$ with $\overline{\mathbf{x}}_{1}^{(k)}(0,0)=0$, and $\mathbf{z}^{(k)}(0,0)=0$ satisfying

$$
\left\{\begin{array}{l}
\frac{\partial \overline{\mathbf{x}}_{1}^{(k)}(v, w)}{\partial v} a(v)=\bar{f}_{1 c}\left(\overline{\mathbf{x}}_{1}^{(k)}(v, w), \mathbf{z}^{(k)}(v, w), v, w\right) \\
\frac{\partial \mathbf{z}^{(k)}(v, w)}{\partial v} a(v)=g_{1} \mathbf{z}^{(k)}(v, w)+g_{2} \mathbf{y}(v, w) \\
\mathbf{y}(v, w)=\bar{h}_{c}\left(\overline{\mathbf{x}}_{1}^{(k)}(v, w), \mathbf{z}^{(k)}(v, w), v, w\right)
\end{array}\right.
$$

In terms of notation $v^{[l]}, \overline{\mathbf{x}}_{1}^{(k)}(v, w), \mathbf{z}^{(k)}(v, w)$ and $\mathbf{y}(v, w)$ can be expressed as

$$
\left\{\begin{array}{l}
\overline{\mathbf{x}}_{1}^{(k)}(v, w)=\sum_{l=1}^{k} \phi_{l w} v^{[l]}+O\left(v^{k+1}\right) \\
\mathbf{z}^{(k)}(v, w)=\sum_{l=1}^{k} \theta_{l w} v^{[l]}+O\left(v^{k+1}\right) \\
\mathbf{y}(v, w)=\sum_{l=1}^{k} Y_{l w} v^{[l]}+O\left(v^{k+1}\right)
\end{array}\right.
$$

Substituting (3.15) into (3.14), and expanding (3.14) as power series in $v^{[l]}, l=$ $1, \cdots, k$, yield the following

$$
\left\{\begin{array}{l}
\phi_{l w} A_{l}=\hat{A}(w) \phi_{l w}+\hat{B}(w) \theta_{l w}+\hat{U}_{l w} \\
\theta_{l w} A_{l}=g_{1} \theta_{l w}+g_{2}\left(\hat{C}(w) \phi_{l w}+\hat{D}(w) \theta_{l w}+\hat{V}_{l w}\right) \\
Y_{l w}=\hat{C}(w) \phi_{l w}+\hat{D}(w) \theta_{l w}+\hat{V}_{l w}
\end{array}\right.
$$

where $\left(\hat{U}_{1 w}, \hat{V}_{1 w}\right)=(\hat{E}(w), \hat{F}(w))$, and for $l=2,3, \cdots,\left(\hat{U}_{l w}, \hat{V}_{l w}\right)$ depends only on $\phi_{1 w}, \cdots, \phi_{(l-1) w}$ and $\theta_{1 w}, \cdots, \theta_{(l-1) w}$.

Equation (3.16) is the Sylvester equation described in Lemma 3.1, and satisfies all conditions of Lemma 3.1. Therefore, it has a unique solution for any $\left(\hat{U}_{l w}, \hat{V}_{l w}\right)$ which satisfies

$$
Y_{l w}=0, l=1, \cdots, k
$$

Thus

$$
\mathbf{y}(v, w)=\bar{h}_{c}\left(\overline{\mathbf{x}}_{1}^{(k)}(v, w), \mathbf{z}^{(k)}(v, w), v, w\right)=O\left(v^{k+1}\right)
$$


Finally define

$$
\left\{\begin{array}{l}
\overline{\mathbf{x}}_{2}^{(k)}(v, w)=\gamma\left(\overline{\mathbf{x}}_{1}^{(k)}(v, w), \mathbf{z}^{(k)}(v, w), w\right) \\
\mathbf{x}^{(k)}(v, w)=T_{2}\left[\overline{\mathbf{x}}_{1}^{(k)}(v, w)^{T}, \overline{\mathbf{x}}_{2}^{(k)}(v, w)^{T}\right]^{T} \\
\mathbf{x}_{c}(v, w)=\left[\mathbf{x}^{(k)}(v, w)^{T}, \mathbf{z}^{(k)}(v, w)^{T}\right]^{T}
\end{array}\right.
$$

Then it is ready to verify that $\mathbf{x}_{c}(v, w)$ satisfies (3.8) upon using (3.14), (3.18), and (3.19).

REMARK 3.3. Consider linear singular system of the form

$$
\left\{\begin{array}{l}
\mathbf{E} \dot{x}=A x+B u+E v \\
y=C x+F v \\
\dot{v}=\hat{A}_{1} v
\end{array}\right.
$$

with $x \in \Re^{n}, u \in \Re^{m}$ and $y \in \Re^{p}$. Assume (3.20) satisfies assumptions A2, A3, and the following

A5:

$$
\operatorname{rank}\left[\begin{array}{cc}
A-\mathbf{E} \lambda & B \\
C & 0
\end{array}\right]=n+p, \forall \lambda \in \sigma\left(\hat{A}_{1}\right) .
$$

Then there exists a controller of form (3.6) in which $g_{1}$, and $g_{2}$ are as described in Lemma 3.1 with $r=p$ and the pair

$$
\left(\left[\begin{array}{cc}
\mathbf{E} & 0 \\
0 & I_{n_{c}}
\end{array}\right],\left[\begin{array}{cc}
A+B K_{2} C & B K_{1} \\
g_{2} C & g_{1}
\end{array}\right]\right)
$$

is strongly stable.

In fact, define

$$
\dot{z}_{1}=G_{1} z_{1}+G_{2} y, \quad z_{1} \in \Re^{n_{G}}
$$

where $\left(G_{1}, G_{2}\right)$ is described as in Lemma 3.1 with $r=p$ with the dimension of $G_{1}$ being denoted by $n_{G}$. Consider the augmented $\left(n+n_{G}\right)$-dimensional system as follows

$$
\left\{\begin{array}{l}
{\left[\begin{array}{cc}
\mathbf{E} & 0 \\
0 & I_{n_{G}}
\end{array}\right]\left[\begin{array}{c}
\dot{x} \\
\dot{z}_{1}
\end{array}\right]=\left[\begin{array}{cc}
A & 0 \\
G_{2} C & G_{1}
\end{array}\right]\left[\begin{array}{c}
x(t) \\
z_{1}
\end{array}\right]+\left[\begin{array}{c}
B \\
0
\end{array}\right] u+\left[\begin{array}{c}
E \\
G_{2} F
\end{array}\right] v} \\
{\left[\begin{array}{c}
y \\
z_{1}
\end{array}\right]=\left[\begin{array}{cc}
C & 0 \\
0 & I_{n_{G}}
\end{array}\right]\left[\begin{array}{c}
x \\
z_{1}
\end{array}\right]+\left[\begin{array}{c}
F \\
0
\end{array}\right] v}
\end{array}\right.
$$

Under assumptions A2, A3, A5 and the fact that $\left(G_{1}, G_{2}\right)$ is controllable, by a known result for linear systems from [8], there exists a control law of the form

$$
\left\{\begin{array}{l}
u=N z_{2}+\left[\begin{array}{ll}
K_{2} & L
\end{array}\right]\left[\begin{array}{c}
y \\
z_{1}
\end{array}\right] \\
\dot{z}_{2}=A_{c} z_{2}+\left[\begin{array}{ll}
B_{c 2} & B_{c 1}
\end{array}\right]\left[\begin{array}{c}
y \\
z_{1}
\end{array}\right]
\end{array}\right.
$$


where $z_{2} \in \Re^{\left(n_{G}+n_{E}\right)}$ such that the closed-loop system is strongly stable. In fact, the controller parameters $N, L$, etc, can be constructed explicitly from Theorem 5-3.2 of [8]. Combining (3.22) and (3.24) gives the controller of the form (3.6) with

$$
z=\left[\begin{array}{c}
z_{2} \\
z_{1}
\end{array}\right], K_{1}=\left[\begin{array}{c}
N \\
L
\end{array}\right], g_{1}=\left[\begin{array}{cc}
A_{c} & B_{c 1} \\
0 & G_{1}
\end{array}\right], g_{2}=\left[\begin{array}{c}
B_{c 2} \\
G_{2}
\end{array}\right]
$$

As a result, we have the following.

COROllary 3.4. Under assumptions A1 to A3, assume the plant composed of (2.1) and (2.2) satisfies A5 with $\hat{A}_{1}=\operatorname{diag}\left\{A_{1}, \cdots, A_{k}\right\}$, then there exists a controller of the form (3.6) such that property P1 holds and equation (3.8) is satisfied.

REMARK 3.5. It can be shown using the center manifold theory [5], [19] that if the closed-loop system satisfies Property P1 and equation (3.8), then for sufficiently small $\left(x_{c 0}, v_{0}\right)$, the solution of the closed-loop system exists for all $t \geq 0$, and is bounded, and

$$
\limsup _{t \rightarrow \infty} y(t)=O\left(v^{k+1}(t)\right), \quad \forall w \in W
$$

For this reason, a controller that renders the closed-loop system these two properties is called $k^{\text {th }}$-order robust regulator.

4. Solvability of the Problem. The $k^{\text {th }}$-order robust regulator is interesting in its own right since it guarantees the steady state tracking error of the closed-loop system is in the order of $k+1$ of the exogenous signal regardless of the small variation of the uncertain parameter $w$. Moreover, under some additional assumptions, the $k^{t h}$-order robust regulator actually solves the robust output regulation problem.

A6: The exosystem is linear, that is, $a(v)=A_{1} v$.

THEOREM 4.1. Under assumptions A1 to A6, and supposing $\mathbf{u}(v, w)$ is $k^{\text {th }}$ degree polynomial in $v$, then the same controller that solves the $k^{\text {th }}$-order robust output regulation also solves the robust output regulation problem.

Proof. By these assumptions, there exists a linear control law of the form (3.6) that solves the $k^{t h}$-order robust regulation problem. Clearly, the closed-loop system composed of the plant and this control law satisfies condition (i) of Theorem 2.2. We need to show that the closed-loop system also satisfies condition (ii) of Theorem 2.2. To this end, consider the following system

$$
\left\{\begin{array}{l}
\mathbf{E} \dot{x}=f\left(\bar{x}, u+K_{2} y, v, w\right) \\
y=h(x, v, w)
\end{array}\right.
$$

Performing the same coordinate transformation on (4.1) as what was done on the closed-loop system (3.9) in the proof of Lemma 3.2 gives

$$
\left\{\begin{array}{l}
\dot{\bar{x}}_{1}=\bar{f}_{1}\left(\bar{x}_{1}, u, v, w\right) \\
y=\bar{h}\left(\bar{x}_{1}, u, v, w\right)
\end{array}\right.
$$


where $\bar{x}_{1}$ is such that $T_{2}^{-1} x=\left(\bar{x}_{1}^{T}, \bar{x}_{2}^{T}\right)^{T}$, and $\bar{f}_{1}\left(\bar{x}_{1}, u, v, w\right)$, and $\bar{h}\left(\bar{x}_{1}, u, v, w\right)$ have the following form

$\bar{f}_{1}\left(\bar{x}_{1}, u, v, w\right)=\hat{A}(w) \bar{x}_{1}+\left(\bar{B}_{1}(w)-\bar{A}_{12}(w) \bar{A}_{22}^{-1}(w) \bar{B}_{2}(w)\right) u+\hat{E}(w) v+o\left(\bar{x}_{1}, z, v, w\right)$

$\bar{h}\left(\bar{x}_{1}, u, v, w\right)=\hat{C}(w) \bar{x}_{1}-C_{2}(w) \bar{A}_{22}^{-1}(w) \bar{B}_{2}(w) u+\hat{F}(w) v+o\left(\bar{x}_{1}, z, v, w\right)$

where all the matrices in the above two equations are defined in the proof of Lemma 3.2. It is easy to verify that

$$
\left\{\begin{array}{l}
\bar{f}_{1}\left(\bar{x}_{1}, K_{1} z, v, w\right)=\bar{f}_{1 c}\left(\bar{x}_{1}, z, v, w\right) \\
\bar{h}\left(\bar{x}_{1}, K_{1} z, v, w\right)=\bar{h}_{c}\left(\bar{x}_{1}, z, v, w\right)
\end{array}\right.
$$

Next, let $\mathbf{x}(v, w)$ and $\mathbf{u}(v, w)$ be the solution of (2.8). Then clearly, $\mathbf{x}(v, w)$ and $\mathbf{u}(v, w)$ also satisfy the following

$$
\left\{\begin{array}{l}
\mathbf{E} \frac{\partial \mathbf{x}(v, w)}{\partial v} A_{1} v=f\left(\mathbf{x}(v, w), \mathbf{u}(v, w)+K_{2} h(\mathbf{x}(v, w), v, w), v, w\right) \\
h(\mathbf{x}(v, w), v, w)=0
\end{array}\right.
$$

Let $T_{2}^{-1} \mathbf{x}(v, w)=\left(\overline{\mathbf{x}}_{1}^{T}(v, w), \overline{\mathbf{x}}_{2}^{T}(v, w)\right)^{T}$. Then (4.2) and (4.4) imply

$$
\left\{\begin{array}{l}
\frac{\partial \overline{\mathbf{x}}_{1}(v, w)}{\partial v} A_{1} v=\bar{f}_{1}\left(\overline{\mathbf{x}}_{1}(v, w), \mathbf{u}(v, w), v, w\right) \\
\bar{h}\left(\overline{\mathbf{x}}_{1}(v, w), \mathbf{u}(v, w), v, w\right)=0
\end{array}\right.
$$

Next we will show that there exists a sufficiently smooth function $\mathbf{z}(v, w)$ such that

$$
\left\{\begin{array}{l}
\mathbf{u}(v, w)=K_{1} \mathbf{z}(v, w) \\
\frac{\partial \mathbf{z}(v, w)}{\partial v} A_{1} v=g_{1} \mathbf{z}(v, w)
\end{array}\right.
$$

To this end, let $\overline{\mathbf{x}}_{1}^{(k)}(v, w)$, and $\mathbf{z}^{(k)}(v, w)$ be as defined in (3.14). Then by (4.3) and (3.18), $\overline{\mathbf{x}}_{1}^{(k)}(v, w)$, and $\mathbf{z}^{(k)}(v, w)$ also satisfy the following equation

$$
\left\{\begin{array}{l}
\frac{\partial \overline{\mathbf{x}}_{1}^{(k)}(v, w)}{\partial v} A_{1} v=\bar{f}_{1}\left(\overline{\mathbf{x}}_{1}^{(k)}(v, w), K_{1} \mathbf{z}^{(k)}(v, w), v, w\right) \\
\bar{h}\left(\overline{\mathbf{x}}_{1}^{(k)}(v, w), K_{1} \mathbf{z}^{(k)}(v, w), v, w\right)=O\left(v^{k+1}\right)
\end{array}\right.
$$

Since $\overline{\mathbf{x}}_{1}^{(k)}(v, w), \mathbf{z}^{(k)}(v, w)$ take the form given by (3.15), and $\phi_{l w}$ and $\theta_{l w}$ satisfy (3.16) and (3.17), comparing (4.5) with (4.7) shows that there exist sufficiently smooth functions $\overline{\mathbf{x}}_{1 k}(v, w)=O\left(v^{(k+1)}\right)$ and $\mathbf{u}_{k}(v, w)=O\left(v^{(k+1)}\right)$ such that

$$
\left\{\begin{array}{l}
\overline{\mathbf{x}}_{1}(v, w)=\sum_{l=1}^{k} \phi_{l w} v^{[l]}+\overline{\mathbf{x}}_{1 k}(v, w) \\
\mathbf{u}(v, w)=\sum_{l=1}^{k} K_{1} \theta_{l w} v^{[l]}+\mathbf{u}_{k}(v, w)
\end{array}\right.
$$

But since $\mathbf{u}(v, w)$ is assumed to be $k^{\text {th }}$ degree polynomial in $v$, it must hold that $\mathbf{u}(v, w)=\sum_{l=1}^{k} K_{1} \theta_{l w} v^{[l]}$. Let $\mathbf{z}(v, w)=\sum_{l=1}^{k} \theta_{l w} v^{[l]}$. Clearly the first equation of (4.6) is satisfied. Now using (3.16) and (3.17) gives $\theta_{l w} A_{l}=g_{1} \theta_{l w}, l=1, \cdots, k$. Hence

$$
\sum_{1}^{k} \theta_{l w} A_{l} v^{[l]}=\sum_{1}^{k} g_{1} \theta_{l w} v^{[l]}
$$


Using $\frac{\partial v^{[l]}}{\partial v} A_{1} v=\dot{v}^{[l]}=A_{l} v^{[l]}$ in (4.8) gives

$$
\frac{\partial \mathbf{z}(v, w)}{\partial v} A_{1} v=\sum_{l=1}^{k} \theta_{l w} \frac{\partial v^{[l]}}{\partial v} A_{1} v=g_{1} \sum_{l=1}^{k} \theta_{l w} v^{[l]}
$$

Thus, the second equation of (4.6) is satisfied.

Finally letting $\mathbf{x}_{c}(v, w)=\left(\mathbf{x}^{T}(v, w), \mathbf{z}^{T}(v, w)\right)^{T}$ and substituting $\mathbf{x}_{c}(v, w)$ and $\mathbf{u}(v, w)$ into (2.5) gives

$$
h_{c}\left(\mathbf{x}_{c}(v, w), v, w\right)=h(\mathbf{x}(v, w), v, w)=0
$$

and, additionally using (2.8) and (4.6) gives

$$
\mathbf{E}_{c} \frac{\partial \mathbf{x}_{c}(v, w)}{\partial v} a(v)=\left[\begin{array}{l}
f(\mathbf{x}(v, w), \mathbf{u}(v, w), v, w) \\
g_{1} \mathbf{z}(v, w)
\end{array}\right]
$$

But (2.5) gives

$$
\begin{aligned}
f_{c}\left(\mathbf{x}_{c}(v, w), v, w\right) & =\left[\begin{array}{c}
f\left(\mathbf{x}(v, w), K_{1} \mathbf{z}(v, w)+K_{2} h(\mathbf{x}(v, w), v, w), v, w\right) \\
g_{1} \mathbf{z}(v, w)+g_{2} h(\mathbf{x}(v, w), v, w)
\end{array}\right] \\
& =\left[\begin{array}{c}
f(\mathbf{x}(v, w), \mathbf{u}(v, w), v, w) \\
g_{1} \mathbf{z}(v, w)
\end{array}\right]
\end{aligned}
$$

Thus condition (ii) of Theorem 2.2 is also satisfied.

REMARK 4.2. In equation (4.1), the introduction of the output feedback term $K_{2} y$ is to modify the system dynamics so that the matrix $\bar{A}_{22}$ as defined in Lemma 3.2 is invertible.

5. Conclusion. This paper has extended the major results of the robust output regulation problem for linear and nonlinear normal systems to nonlinear singular systems. Even though only the sufficient conditions are given here, it is possible to establish the necessary conditions for the existence of a $k^{\text {th }}$-order order robust regulator. In fact, assume the variations of $w$ are such that each entry of $(E(w), F(w))$ can vary arbitrarily in a neighborhood of the origin, then using the argument similar to what was used for normal nonlinear systems [12], it can be shown that, assumption A5 is also the necessary condition for the existence of the $k^{t h}$-order robust output regulator.

\section{REFERENCES}

[1] J. D. Aplevich, Implicit Linear Systems, Springer-Verlag, New York, 1981.

[2] C. I. Byrnes, Priscoli F. Delli, A. Isidori, And W. Kang, Structurally stable output regulation of nonlinear systems, Automatica, 33(1997), pp. 369-385.

[3] S. L. Campbell and E. Griepentrog, Solvability of general differential algebraic equations, SIAM Journal of Science and Computing, 16(1995), pp. 257-270.

[4] S. L. Campbell, Singular systems of differential equations, Pitman, New York, 1980. 
[5] J. CARR, Applications of the Center Manifold Theory, Springer Verlag, 1981.

[6] D. СовB, Controllability, observability and duality in singular systems, IEEE Tran. on Automatic Control, 26(1984), pp. 1076-1082.

[7] Z. L. Cheng And J. F. Zhang, Strong controllability, Stabilizability, pole-placement and inputoutput decoupling of generalized systems, Proceedings of Systems and Control, pp 88-94, Shandong University Press, 1986.

[8] L. Y. DAI, Singular control systems, Lecture Notes in Control and Information Sciences, V.118, Springer-Verlag, New York, 1989.

[9] E. J. DAvison, The robust control of a servomechanism problem for linear time-invariant multivariable systems, IEEE Tran. on Automatic Control, V.AC-21:1(1976).

[10] B. A. Francis, The Linear Multivariable Regulator Problem, SIAM Journal on Control and Optimization, 15(1977), pp. 486-505.

[11] B. A. Francis and W. M. Wonham, The Internal Model Principle of Control Theory, Automatica, 12(1976), pp. 457-465.

[12] J. HuANG, K-fold exosystem and the robust nonlinear servomechanism problem, ASME Journal of Dynamics, Control, and Measurement, 1996, pp. 149-153.

[13] J. Huang And C. F. Lin, On a robust nonlinear servomechanism problem, IEEE Tran. on Automatic Control, 39:7(1991).

[14] J. Huang And C. F. Lin, Internal Model Principle and Robust Control of Nonlinear Systems, Proceedings of the 32nd Conference on Decision and Control, 1993.

[15] J. HuAng And C. F. Lin, On the solvability of the general nonlinear servomechanism problem, Proceedings of American Control Conference, June 1993, pp. 2958-2959.

[16] J. HuAng AND W. J. Rugh, On a nonlinear multivariable servomechanism problem, Automatica, 26(1990), pp. 963-972.

[17] J. HuAng AND W. J. Rugh, Stabilization on zero-error manifolds and the nonlinear servomechanism problem, IEEE Tran. on Automatic Control, 37(1992), pp. 1009-1013.

[18] J. HuAng AND J. F. Zhang, Impulse-free output regulation of singular nonlinear systems, Int. Journal of Control, 71:5(1998), pp. 789-806.

[19] A. Isidori And C. I. Byrnes, Output regulation of nonlinear systems, IEEE Tran. on Automatic Control, 35:2(1990).

[20] F. L. LEWIS, Fundamental, reachabilty and observability matrices of discrete descriptor systems, IEEE Tran. on Automatic Control, 30(1985), pp. 502-505.

[21] W. Lin AND L. Y. DAI, Solutions to the output regulation problem of linear singular systems, Automatica, 32(1996), pp. 1713-1718.

[22] G. C. Verghese, B. C. Levy, and L. Kailath, A generalized state-space for singular systems, IEEE Tran. on Automatic Control, 26(1981), pp. 811-831.

[23] E. L. Yip AND R. F. Sincovec, Solvability, controllability and observability of continuous descriptor systems, IEEE Tran. on Automatic Control, 26(1981), pp. 702-707. 